\title{
ON A GENERALIZED DIVISOR PROBLEM II
}

\author{
YUK-KAM LAU
}

\begin{abstract}
We investigate the $\Omega_{ \pm}$-result of $\Delta_{a}(x)$ and its number of signchanges in an interval $[1, T]$, denoted by $X_{a}(T)$, for $-1 \leq a<-1 / 2$. We can prove that $T \ll{ }_{a} X_{a}(T)$ which is the best possible in order of magnitude.
\end{abstract}

\section{$\S 1$. Introduction}

Let $-1<a<0$ and define

$$
\Delta_{a}(x)=\sum_{n \leq x} \sigma_{a}(n)-\zeta(1-a) x-\frac{\zeta(1+a)}{1+a} x^{1+a}+\frac{1}{2} \zeta(-a)
$$

where $\sigma_{a}(n)=\sum_{d \mid n} d^{a}$. The case $a=-1$ is defined by taking the righthand limit. Here, we do not half the last term in the sum when $x$ is an integer, in order to match the definition of some authors and to help simplifying later calculations. As was discussed in [4], the behaviour of $\Delta_{a}(x)$ for $-1 \leq a<-1 / 2$ is different from the case $-1 / 2<a \leq 0$ and $a=-1 / 2$ appears as a critical point. Furthermore, we find in [5] that the limiting distribution for the case $-1 \leq a<-1 / 2$ is symmetric while the case $a=0$ is not. This further supports the change in nature. Therefore, we want to explore more properties of $\Delta_{a}(x)$ in these two ranges in order to realize their differences.

In [4], we investigated the oscillatory nature of $\Delta_{a}(x)$ for $-1 / 2 \leq a<0$. In this paper, we continue our study for the other case by considering the extreme values and the number of sign-changes of $\Delta_{a}(x)$. Certainly, large extreme values show a great amplitude of fluctuation and plenty of signchanges tell us that it is very oscillatory.

Through the mean square formula (see [4, Section 1]), we expect that $\Delta_{a}(x) \ll x^{\epsilon}$ when $-1 \leq a<-1 / 2$. The theorem below gives a result in the opposite direction.

Received March 21, 2001.

2000 Mathematics Subject Classification: 11N64. 
ThEOREM 1. We have, for $-1<a \leq-1 / 2$,

$$
\Delta_{a}(x)=\Omega_{ \pm}\left(\exp \left((1+o(1)) \frac{1}{1-|a|}\left(\frac{|a|}{2}\right)^{1-|a|} \frac{(\log x)^{1-|a|}}{\log \log x}\right)\right)
$$

and $\Delta_{-1}(x)=\Omega_{ \pm}(\log \log x)$.

This result, which seems to be the sharpest to date, was obtained by Pétermann [9] for $-1 \leq a<-25 / 38$. We extend the range of $a$ up to $-1 / 2$ by his argument together with a simple idea, which is the use of an averaged result of $G_{|a|}(x)$ (in Lemma 2.3) instead of a bound derived by the method of exponent pairs.

Concerning with the sign-changes of $\Delta_{a}(x)$, Pétermann studied this problem as well and he obtained in [6] that

$$
X_{a}(T) \geq \frac{8}{3}\left(1-\frac{\zeta(2|a|)}{4 \zeta(2+2|a|)}\right) T+o(T)
$$

where $X_{a}(T)$ denotes the number of sign-changes of $\Delta_{a}(x)$ in $[1, T]$. (Here, a sign-change of a function $f$ at $x_{0}$ means $f\left(x_{0}-\right) f\left(x_{0}+\right)<0$.) It should be remarked that (i) $X_{a}(T) \ll T$ and (ii) the main term in (1.2) is positive only when $a<-0.6236622010 \ldots$ It is apparent that $\Delta_{a}(x)$ decreases by an amount of $-\zeta(1-a)+o(1)($ as $n \rightarrow \infty)$ when $x$ varies over $[n, n+1)$ where $n$ is an integer. The sign-changes counted in $X_{a}(T)$ may be due to the fact that plenty of $\Delta_{a}(n)(n \in \mathbb{N})$ just lie above the $x$-axis. This leads to the consideration of sign-changes at integral points. In [2] and [8], problem of this type has been studied for the Euler Phi function. Let us denote by $N_{a}(T)$ the number of sign-changes on integers (i.e. $\Delta_{a}(n) \Delta_{a}(n+1)<0$ with $n \in \mathbb{N})$. Clearly, the determination of $N_{a}(T)$ is harder and it was shown in [8] that $N_{-1}(T) \gg T^{0.71468244}$.

Our next result can extend the range of $a$ in (1.2) to $-1 / 2$ and this shows the consistency in oscillatory behaviour of $\Delta_{a}(x)$ for $a \in[-1,-1 / 2)$. (Note that the case $a=-1 / 2$ is not included.) Moreover, it yields a lower bound for $N_{-1}(T)$. Let us say that a real-valued function $f(x)$ has a signchange behind an integer $n$ if $f(n) f(n+r)<0$ and

$$
f(n+1)=f(n+2)=\cdots=f(n+r-1)=0
$$

for some natural number $r$ (independent of $n$ ). Then we have 
Theorem 2. Let $N_{a, 0}(T)=\operatorname{Card}\left\{n \in[1, T]: \Delta_{a}(x)\right.$ has a sign-change behind $n\}$. Then, $N_{a, 0}(T) \gg_{a} T$ for $-1 \leq a<-1 / 2$ and all sufficiently large $T$.

An immediate consequence is $X_{a}(T) \gg T$ for $-1 \leq a<-1 / 2$ by looking at the graph of $\Delta_{a}(x)$. Another consequence is an improvement of the lower bound for $N_{-1}(T)$, which is best possible in order of magnitude.

Corollary. We have $N_{-1}(T) \gg T$.

\section{§2. Proof of Theorem 1}

To prove Theorem 1, we need some lemmas. Lemma 2.1 is our basic tool. By using it, we obtain Lemmas 2.2 and 2.3 which rely on the arguments in [7] and [9].

Lemma 2.1. For $-1 \leq a \leq-1 / 2$, let $\psi(u)=u-[u]-1 / 2$ where $[u]$ is the integral part of $u$,

$$
\Delta_{a}(t)=-\sum_{n \leq \sqrt{t}} n^{a} \psi\left(\frac{t}{n}\right)-t^{a} \sum_{n \leq \sqrt{t}} n^{|a|} \psi\left(\frac{t}{n}\right)+O\left(t^{a / 2}\right) .
$$

For the case $-1<a \leq-1 / 2$, it was proved in Chowla [1, Lemma 15] but, in fact, the argument applies to the case $a=-1$ as well.

Define $G_{a}(x)=\sum_{n \leq \sqrt{x}} n^{a} \psi(x / n)$. Then one can find the following result in [7] or [9]. We include a proof here as it helps us to prove the next lemma.

Lemma 2.2. Let $A$ be a squarefree integer and $B$ be an integer with $|B| \leq A-1$. For $-1 \leq a \leq-1 / 2$, we have

$$
\frac{1}{X} \sum_{m \leq X} G_{a}(A m+B)=\sum_{n \leq \sqrt{A X+B}}(A, n) n^{a-1} \psi\left(\frac{B}{(A, n)}\right)+O\left(A(A X)^{a / 2}\right)
$$

where $(A, n)$ is the greatest common divisor of $A$ and $n$. In particular,

$$
\begin{aligned}
& \frac{1}{X} \sum_{m \leq X} G_{a}(A m)=-\frac{1}{2} \zeta(1-a) \prod_{p \mid A}\left(1+p^{a}-p^{a-1}\right)+O\left(A(A X)^{a / 2}\right), \\
& \frac{1}{X} \sum_{m \leq X} G_{a}(A m-1)=\frac{1}{2} \zeta(1-a) \prod_{p \mid A}\left(1+p^{a}-p^{a-1}\right) \\
&-\zeta(1-a)+O\left(A(A X)^{a / 2}\right) .
\end{aligned}
$$


Proof. Let $n^{*}=n /(A, n)$. Then it is not difficult to see that

$$
\begin{aligned}
\sum_{u \leq m \leq v} \psi\left(\frac{A m+B}{n}\right) & =\frac{v-u}{n^{*}} \sum_{m=0}^{n^{*}-1} \psi\left(\frac{m}{n^{*}}+\frac{B}{n}\right)+O\left(n^{*}\right) \\
& =\frac{v-u}{n^{*}} \psi\left(\frac{B}{(A, n)}\right)+O\left(n^{*}\right) .
\end{aligned}
$$

(See [7, Lemma 1] for details.) From the definition of $G_{a}(x)$ and (2.1),

$$
\begin{aligned}
& \text { 2) } \frac{1}{X} \sum_{m \leq X} G_{a}(A m+B) \\
& =\frac{1}{X} \sum_{n \leq \sqrt{A X+B}} n^{a}\left(\left(X-\max \left(1, \frac{n^{2}-B}{A}\right)\right) \frac{1}{n^{*}} \psi\left(\frac{B}{(A, n)}\right)+O\left(n^{*}\right)\right) \\
& =\sum_{n \leq \sqrt{A X+B}}(A, n) n^{a-1} \psi\left(\frac{B}{(A, n)}\right) \\
& \quad-X^{-1} \sum_{n \leq \sqrt{A X+B}}(A, n) n^{a-1} \max \left(1, \frac{n^{2}-B}{A}\right) \psi\left(\frac{B}{(A, n)}\right) \\
& \quad O\left(X^{-1} \sum_{n \leq \sqrt{A X+B}} n^{a+1}\right) .
\end{aligned}
$$

The $O$-term is obviously $\ll A(A X)^{a / 2}$ and the second sum in $(2.2)$ is

$$
\begin{aligned}
& \ll(A X)^{-1} \sum_{n \leq \sqrt{A X}}(A, n) n^{a+1}+A X^{-1} \\
& \ll(A X)^{-1} \sum_{d \mid A} d^{a+2} \sum_{n \ll \sqrt{A X} / d} n^{a+1}+A X^{-1} \\
& \ll(A X)^{a / 2} \sigma_{0}(A)+A X^{-1} \ll A(A X)^{a / 2} .
\end{aligned}
$$

This yields the first part of Lemma 1 .

When $B=0$, we have $\psi(0)=-1 / 2$ and the first sum in (2.2) is equal 
to

$$
\begin{aligned}
& -\frac{1}{2} \sum_{n \leq \sqrt{A X}} n^{a-1}(A, n) \\
= & -\frac{1}{2} \sum_{d \mid A} d^{a} \sum_{\substack{n=1 \\
(n, A / d)=1}}^{\infty} n^{a-1}+O\left(A(A X)^{a / 2}\right) \\
= & -\frac{1}{2} \zeta(1-a) \prod_{p \mid A}\left(1+p^{a}-p^{a-1}\right)+O\left(A(A X)^{a / 2}\right) .
\end{aligned}
$$

The case $B=-1$ follows by similar argument with $\psi(-1 /(A, n))=1 / 2-$ $1 /(A, n)$.

Lemma 2.3. Let $-1 \leq a \leq-1 / 2$. For $B=0$ or -1 , we have

$$
\frac{1}{X} \sum_{m \leq X}(A m+B)^{a} G_{|a|}(A m+B) \ll(A X)^{a / 2}\left(A+(A X)^{\epsilon}\right)
$$

where $A$ is a squarefree integer.

Proof. Consider the case $B=0$, we have, from (2.1), $\sum_{u \leq m \leq v} \psi\left(\frac{A m}{n}\right) \ll$ $(v-u) / n^{*}+n^{*}$. This yields

$$
\sum_{n^{2} / A \leq m \leq X} m^{a} \psi\left(\frac{A m}{n}\right) \ll \frac{X^{a+1}}{n^{*}}+\left(\frac{n^{2}}{A}\right)^{a} n^{*} .
$$

Then,

$$
\begin{aligned}
& X^{-1} \sum_{m \leq X}(A m)^{a} G_{|a|}(A m) \\
= & A^{a} X^{-1} \sum_{n \leq \sqrt{A X}} n^{|a|} \sum_{n^{2} / A \leq m \leq X} m^{a} \psi\left(\frac{A m}{n}\right) \\
\ll & A^{a} X^{-1} \sum_{n \leq \sqrt{A X}} n^{|a|}\left(\frac{X^{a+1}}{n^{*}}+\left(\frac{n^{2}}{A}\right)^{a} n^{*}\right) \\
\ll & (A X)^{a} \sum_{n \leq \sqrt{A X}} n^{|a|-1}(A, n)+X^{-1} \sum_{n \leq \sqrt{A X}} n^{a+1} \\
\ll & \sigma_{0}(A)(A X)^{a / 2}+A(A X)^{a / 2}
\end{aligned}
$$


by using the argument in (2.3).

To prove the case $B=-1$, it suffices to check that $G_{|a|}(A m)-G_{|a|}(A m-$ $1) \ll(A m)^{|a| / 2+\epsilon}$. This follows from the observation that if $n$ does not divide $A m$,

$$
\psi\left(\frac{A m}{n}\right)-\psi\left(\frac{A m-1}{n}\right)=\frac{1}{n}
$$

and $\ll 1$ otherwise.

Proof of Theorem 1. Taking $A=\prod_{2<p \leq y} p$ where $y$ is chosen such that $A \asymp X^{|a| /(2+a)}$, by Lemmas 2.2 and 2.3 , we have immediately that

$$
X^{-1} \sum_{m \leq X} \Delta_{a}(A m)=\frac{1}{2} \zeta(1-a) \prod_{p \mid A}\left(1+p^{a}-p^{a-1}\right)+O\left(A(A X)^{a / 2}\right)
$$

and

$$
\begin{aligned}
& X^{-1} \sum_{m \leq X} \Delta_{a}(A m-1) \\
= & -\frac{1}{2} \zeta(1-a) \prod_{p \mid A}\left(1+p^{a}-p^{a-1}\right)+\zeta(1-a)+O\left(A(A X)^{a / 2}\right) .
\end{aligned}
$$

The value of $\prod_{2<p \leq y}\left(1+p^{a}-p^{a-1}\right)$ is equal to

$$
\begin{aligned}
& \exp \left(\sum_{2<p \leq y} p^{a}+O\left(\sum_{2<p \leq y} p^{2 a}\right)\right) \\
= & \begin{cases}\exp \left((1+o(1)) y^{1+a} /((1+a) \log y),\right. & \text { if }-1<a \leq-1 / 2 \\
\exp (\log \log y+O(1)) & \text { if } a=-1,\end{cases}
\end{aligned}
$$

by the Prime Number Theorem. Observing that $\sup _{1 \leq u \leq A X} \Delta_{a}(u) \geq X^{-1}$ $\sum_{m \leq X} \Delta_{a}(A m)\left(\right.$ and $\sup _{1 \leq u \leq A X}\left(-\Delta_{a}(u)\right) \geq-X^{-1} \sum_{m \leq X} \Delta_{a}(A m-1)$ ), our result follows after replacing $A X$ by $x$. Noting that $x \asymp X^{2 /(2+a)} \asymp$ $e^{2 y /|a|}$, we have $y=(|a| \log x) / 2+O(1)$.

\section{$\S 3$. Proof of Theorem 2 and Corollary}

Our approach is to show that there are many integers at which $\Delta_{a}(x)$ takes negative values. From the definition, we see that the graph of $\Delta_{a}(x)$ is essentially a straight line of negative slope on each interval $[n, n+1)$. If 
the number of integers $n$ satisfying $\Delta_{a}(n)>0$ is small, then the absolute value of the integral of $\Delta_{a}(x)$ should be large since the positive area cannot give much cancellation to the negative. We find that it is not the case and hence Theorem 2 can be proved. (This method can be applied to the case of the Euler Phi function as well.)

To complete the first task, we consider the distribution functions. Let $P_{a, X}(u)=X^{-1} \operatorname{Card}\left\{1 \leq n \leq X: \Delta_{a}(n) \leq u\right\}$ and $D_{a, X}(u)=X^{-1} \mu\{t \in$ $\left.[1, X]: \Delta_{a}(t) \leq u\right\}$ where Card means the cardinality and $\mu$ is the Lebesgue measure. We have

$$
D_{a, X}(u-\zeta(1-a)) \leq P_{a, X}(u) \leq D_{a, X}(u)+O\left(X^{-1}\right) .
$$

(This can be seen as follows: by (1.1), for any $t \in[n, n+1)$,

$$
\Delta_{a}(n)-\Delta_{a}(t)=(t-n)\left(\zeta(1-a)+\zeta(1+a) \xi^{a}\right)
$$

for some $\xi \in(n, t)$. As $\zeta(1+a)<0$ for $-1<a<0, \Delta_{a}(t) \leq \Delta_{a}(n)$ for all sufficiently large $n$. This yields the right side. Also, it follows that $\Delta_{a}(n) \leq$ $\Delta_{a}(t)+\zeta(1-a)$ and hence the left side of (3.1).) From [5, Theorem 3], we see that $D_{a}(u)=\lim _{X \rightarrow \infty} D_{a, X}(u)$ is a symmetric (i.e. $1-D_{a}(u)=D_{a}(-u)$ ) probability distribution function. Moreover, we can prove

Lemma 3.1. For all real $u, 0<D_{a}(u)<1$.

Proof. As a distribution function is increasing, it suffices to show $D_{a}(-u)>0$ for all sufficiently large $u$. Let $u$ be any large number, and define $y$ by the equations $\log u=y^{1+a} / \log y$ if $-1<a<-1 / 2$ or $u=\log y$ if $a=-1$. Write $A=\prod_{2 \leq p \leq y} p$, then $\sigma_{a}(A)=\prod_{p \mid A}\left(1+p^{a}\right) \gg u$ (see $(2.5))$. Since $\sigma_{a}(A m) \geq \sigma_{a}(\bar{A})$ for any integer $m$, we get

$$
\Delta_{a}(A m)-\Delta_{a}(A m-1)=\sigma_{a}(A m)+O(1) \gg u .
$$

This implies $\left|\Delta_{a}(A m)\right| \gg u$ or $\left|\Delta_{a}(A m-1)\right| \gg u$; hence

$$
1-P_{a, X}(u)+P_{a, X}(-u)=X^{-1} \operatorname{Card}\left\{1 \leq n \leq X:\left|\Delta_{a}(n)\right| \geq u\right\} \gg A^{-1} .
$$

Using (3.1) and taking $X \rightarrow \infty$, we deduce that

$$
1-D_{a}(u-\zeta(1-a))+D_{a}(-u) \gg A^{-1}>0 .
$$

Replacing $u$ by $u+\zeta(1-a)$ and observing that $D_{a}(-u) \geq D_{a}(-u-\zeta(1-$ a)) (since $\zeta(1-a)>0)$, we conclude with the symmetry of $D_{a}(u)$ that $2 D_{a}(-u)=1-D_{a}(u)+D_{a}(-u)>0$. Our proof is then complete.

The next lemma is to show that the integral of $\Delta_{a}(x)$ is small on average. 
Lemma 3.2. Let $-1 \leq a<-1 / 2$ and $1 \ll h \ll \sqrt{T}$. Define $E_{a}(t)=$ $\int_{0}^{t} \Delta_{a}(v) d v$. We have

$$
\int_{T}^{2 T}\left(E_{a}(t+h)-E_{a}(t)\right)^{2} d t \ll T h^{3+2 a} \min \left((1-|a|)^{-1}, \log h\right) .
$$

Proof. From [3], we can establish a (truncated) Voronoi-type formula for $E_{a}(t)$. This is obtained by taking $\delta=1+a, \rho=1, \phi(s)=\psi(s)=$ $\pi^{-s} \zeta(s) \zeta(s-a)$ and $\Delta(s)=\Gamma(s / 2) \Gamma((s-a) / 2)$ there. Then we see that $a_{n}=b_{n}=\sigma_{a}(n), \lambda_{n}=\mu_{n}=\pi n, A=1, h=4, k_{0}(\rho)=-3 / 4, e_{0}(\rho)=$ $1 /(2 \sqrt{2 \pi})$ and $\theta_{\rho}=3 / 4+a / 2$ as $\rho=1$. Noting that $E_{a}(t)=\pi^{-1} E_{A, 1}(\pi t)$, we apply [3, Theorem 1] with $X=2 T, Z=4 T$ and observe that the second sum is $\ll T^{-1 / 2} \sum_{n<4 T} \sigma_{a}(n) n^{-(3 / 4+a / 2)} \min \left(1,|t-n|^{-2}\right) \ll 1$. We get for $t \in[T, 2 T]$,

$$
E_{a}(t)=\frac{1}{2 \sqrt{2} \pi^{2}} t^{3 / 4+a / 2} \sum_{n \leq 4 T} \frac{\sigma_{a}(n)}{n^{5 / 4+a / 2}} w_{T}(n) \cos \left(4 \pi \sqrt{n t}-\frac{3 \pi}{4}\right)+O(1)
$$

where $w_{T}(u)=1$ for $1 \leq u \leq 2 T$ and $w_{T}(u)=2-u /(2 T)$ for $2 T \leq u \leq 4 T$. Then Lemma 3.2 is complete with the argument in [4, Theorem 1].

Proof of Theorem 2. Let $\epsilon>0$ be a small fixed number. By Lemma 3.1, $D_{a}(-\epsilon-\zeta(1-a))$ is a positive constant. Hence, for all sufficiently large $T$, we have from $(3.1)$ that $P_{a, T}(-\epsilon) \geq D_{a, T}(-\epsilon-\zeta(1-a)) \geq \kappa$ for some positive constant $\kappa$ depending on $a$ and $\epsilon$. Let $H$ be a large number which will be chosen later. Cutting the interval $[T, 2 T]$ into subintervals of length $H$, there are at least $\kappa T / H-O(1)$ subintervals, each of which contains an integer $n$ such that $\Delta_{a}(n)<-\epsilon<0$. We can then form a class $\mathcal{C}$ from these subintervals such that (i) the cardinality of $\mathcal{C} \geq \kappa T /(4 H)$, (ii) any two intervals in $\mathcal{C}$ is separated by a distance not less than $2 H$, and (iii) for any $I \in \mathcal{C}, \Delta_{a}(n)<0$ for some $n \in I$. This can be done by picking one from every three consecutive subintervals.

Now, we single out the interval $I \in \mathcal{C}$ which has the following property: there is $n_{I} \in I$ such that $\Delta_{a}(m) \leq 0$ for all $n_{I} \leq m \leq n_{I}+2 H$. Let $M$ be the number of such intervals. When $\Delta_{a}(m) \leq 0, \int_{m}^{m+1} \Delta_{a}(u) d u \leq$ $-\zeta(1-a) / 2+O\left(m^{a}\right)$. Then, we have for all real $t \in\left[n_{I}, n_{I}+H\right]$,

$$
\left|E_{a}(t+H)-E_{a}(t)\right|=\left|\int_{t}^{t+H} \Delta_{a}(u) d u\right| \gg_{a} H
$$


Hence $\int_{n_{I}}^{n_{I}+H}\left(E_{a}(t+H)-E_{a}(t)\right)^{2} d t \gg H^{3}$ and this yields $M H^{3} \ll \int_{T}^{2 T}\left(E_{a}(t\right.$ $\left.+H)-E_{a}(t)\right)^{2} d t$. By Lemma 3.2, $M \ll T H^{2 a} \log H$. We select a large constant $H$ so that $\kappa T /(4 H)-O\left(T H^{2 a} \log H\right) \gg_{a} T$. Therefore there are $\gg_{a} T$ subintervals, in which there is an integer $n$ satisfying $\Delta_{a}(n)<0$ but $\Delta_{a}(m)>0$ for some integer $m$ in $[n+1, n+H]$. This completes the proof.

At last, we prove the corollary. It follows from the fact that the limiting distribution $P_{-1}(u)=\lim _{X \rightarrow \infty} P_{-1, X}(u)$ is a continuous function (see the last section of [8]). Hence the number of $n \in[1, T]$ such that $\Delta_{-1}(n)$ equals zero is $o(T)$. This means that $N_{-1}(T)=N_{-1,0}(T)+o(T)$ and the result follows.

\section{REFERENCES}

[1] S. Chowla, Contributions to the analytic theory of numbers, Math. Z., 35 (1932), 279-299.

[2] P. Erdös and H.M. Shapiro, On the changes of sign of a certain error function, Canada. J. Math., 3 (1951), 375-385.

[3] Y. -K. Lau, On the mean square formula of the error term for a class of arithmetical function, Mh. Math., 128 (1999), 111-129.

[4] - On a generalized divisor problem I, Nagoya Math. J., 165 (2002), 71-78.

[5] $\longrightarrow$ On the limiting distribution of a generalized divisor problem for the case $-1 \leq a<-1 / 2$, Acta Arith., 98 (2001), 237-244.

[6] Y. -F. S. Pétermann, Changes of sign of error terms related to Euler's function and to divisor functions, Comm. Math. Helv., 61 (1986), 84-101.

[7] — About a Theorem of Paolo Codecà's and $\Omega$-Estimates for Arithmetical Convolutions, J. Number Theory, 30 (1988), 71-85.

[8] On the distribution of values of an error term related of the Euler functions, Théorie des nombres, (Quebec, PQ, 1987), de Gruyter, Berlin (1989), pp. 785-797.

[9] About a Theorem of Paolo Codecà's and Omega Estimates for Arithmetical Convolutions, Second Part, Ann. Scuola Norm. Sup. Pisa Cl. Sci., (4) 17 (1990), 343-353.

Institut Élie Cartan

Université Henri Poincaré (Nancy 1)

54506 Vandoeuvre lés Nancy Cedex, France

CURRENT ADDRESS

Department of Mathematics

The University of Hong Kong

Pokulam Road, HONG KONG

yklau@maths.hku.hk 